\title{
The Search for Ourselves in the Universe: A Review of Contingency and Convergence: Toward a Cosmic Biology of Body and Mind
}

\author{
Letitia Meynell*
}

\begin{abstract}
Russell Powell's (2020) new book, Contingency and Convergence: Toward a Cosmic Biology of Body and Mind, argues that universal biological laws, evidenced by convergent evolution, produce humanlike consciousness, not just on Earth but throughout the universe, while maintaining that aspects of human cognition are nonetheless unique. Drawing from Stephen Jay Gould's account of the contingency of evolution, against which he contrasts his view, I argue that Powell exemplifies a troubling anthropocentrism that Gould flatly denounces. Further, I argue that this anthropocentrism leads Powell to make implausible claims about other animal minds and the likelihood of human-like consciousness on planets other than our own.
\end{abstract}

\section{Keywords}

anthropocentrism $\bullet$ biological laws $\bullet \operatorname{cognition} \bullet$ contingency $\bullet$ convergence $\bullet$ evolution $\bullet$ minds $\bullet$ nonhuman animals $\bullet$ Gould, Stephen Jay $\bullet$ Umwelts $\bullet$ vision

The basic thesis of Russell Powell's (2020) Contingency and Convergence: Toward a Cosmic Biology of Body and Mind is that law-like evolutionary processes produce humanlike cognitive capacities (even consciousness), rendering such capacities common in the universe. There is an important caveat; key aspects of human cognition, those that undergird cumulative culture, are entirely contingent and likely very rare. To defend this thesis, Powell marshals a wealth of evidence from a variety of disciplines and develops some singular theoretical tools. Unfortunately, at a number of key points in the book Powell simply ignores contradicting evidence and other plausible approaches to thinking about the history of life on our planet. Thus Powell's argument is, at best, a weak how possibly argument-another Just-So story mixing science with speculation. Nonetheless, the flaws in the book are instructive. They exemplify how a kind of anthropocentrism continues to shape the philosophy of biology and, arguably, biology itself, placing humans at the apex of evolution and demanding that the study of life must ultimately be about us. In

*Department of Philosophy and the Gender and Women's Studies Programme, Dalhousie University,

Halifax, NS B3H 4R2, Canada, Letitia.Meynell@Dal.Ca

Received 9 August 2020; Revised 10 February 2021; Accepted 12 February 2021

doi:10.3998/ptpbio.16039257.0013.004

๑ OPEN ACCESS - PTPBIO.ORG 
effect, Powell offers a rationalization for why we can treat humans as the measure of all things, asserting our continuity with the rest of life on the planet, while at the same time maintaining our uniqueness. Interrogating his argument proves a useful exercise in identifying why this kind of anthropocentrism is implausible (and unpalatable).

That said, there is some excellent scholarship in the book and it is a pleasure to read something that is bold and wide ranging. Powell is addressing the question of the ubiquity and character of life in the universe-perhaps the most general issue a philosopher of biology can tackle-and approaches the topic fearlessly.

Powell's motivating question is "Are we alone in the universe?" (13). Juxtaposed with the introductory chapter's epigraph—a quote by Carl Sagan that identifies the project of the life sciences and exobiology in particular with "the search for who we are" (1), this question suggests the anthropocentric project of the book. However, a suitable troubling of what is meant by the "we" in "Are we alone" (13) allays initial suspicions, as does the approach that Powell initially takes. Like others who have tackled this question, Powell reframes it as whether relevant biological phenomena on our planet "can reliably be projected onto other life worlds" (14). To answer this, Powell maintains we need a "conceptual framework for separating law-like from accidental features of the evolutionary process" (14). Convergent evolution is presented as the answer to this need, providing evidence of projectable regularities, which reveal universal biological laws. With this framing, Powell enters a longstanding debate between Stephen Jay Gould and his critics about what would happen if we could go back in time and "replay the tape of life."

Part I of the book attempts to unpack Gould's position, situating it in conversation with his interlocutors, and identifying robust criteria for true convergent evolution. The goal is to identify the two conflicting extremes so that Powell can occupy a middle ground that is sensitive to Gould's insights but nonetheless reveals the existence of universal biological laws. In Chapter Two, Powell describes Gould's provocative thought experiment. Gould takes us back to the Cambrian explosion, preserved in the Burgess Shale, and the extraordinary diversity of fauna that has been found there. These animals, most of which were wiped out during one of the many mass extinction events that pepper the history of life, had a wide variety of different body plans constituting many different phyla. Although it is tempting to think that the handful of surviving contemporary animal phyla - such as arthropods, molluscs and our own, chordatesmust have been in some sense better adapted, Gould argues that there is no real evidence to support this. They were just lucky. Contemporary species, including our own, are just the beneficiaries of an evolutionary lottery. In Gould's $(1990,25)$ words, "The history of life is a story of massive removal followed by differentiation within a few surviving stocks, not the conventional tale of steadily increasing excellence, complexity, and diversity." So, if we were to go back to the Cambrian and replay the tape of life with slightly different initial conditions, neither humans nor anything like us might ever have evolved. Importantly, this is because the body plans that evolved in the Cambrian produced developmental constraints that significantly limit the morphologies, physiologies, and capacities that could evolve in their descendants.

Contra Gould, a number of people have argued that if we were to replay the tape of life, even from very different initial conditions, something very much like us, if not exactly human, would have evolved. Fundamentally at issue is the question of whether natural history is like history - a contingent set of events that, though typically explicable after the fact, are impossible to predict - or whether natural history, like cosmic history, is governed by laws of nature (or something like them) that are projectable and can provide robust predictions.

Having drawn the contours of the debate, Powell tackles what is meant by biological laws and, concomitantly, robustly replicable outcomes along with their antithesis, radically contingent outcomes. Radical contingency is given a clear definition- "Outcome $O$ is radically contingent 
if and only if a marginal change in some initial condition $I_{1}, \ldots, I_{n}$ would tend to result in outcome $O^{*}$ in any physically possible world, where $O^{*}$ is radically disparate from $O$ " (75). Robust replicability and law-likeness remain vague. Natural selection is clearly central to robust replicability and Powell asserts that there is a "strong [case] to be made that the principles of natural selection ... are ... laws in the strict sense" (83). However, he does not explain or defend this dubious claim (simply citing Brandon's work on the topic). Anyone taken with Peter Godfrey-Smith's (2009, 17-40, esp. 30-31) account of natural selection as a rough recipe with a "patchwork" of empirically tractable models will be unimpressed.

Seemingly alert to his readers' possible scepticism, Powell assures us that ultimately the debate rests "on whether there are nontrivial bundles of specific properties that, like their celestial counterparts, exhibit a truly robust range of counterfactual stability" (87). This, however, does little to assuage doubts. It is difficult to take the comparison with "celestial counterparts" particularly seriously. It is surely fanciful to suppose that natural selection would produce counterfactually stable phenomena as robust as the relation between hydrogen, mass, gravity, and nuclear fusion that powers stars. One of the best examples of convergent evolution-the nontrivial bundle of spine, fusiform, and aquatic environments that has shaped the dolphin, the shark, and the ichthyosaur-has countless exceptions, from eels, to skates, to boxfish. Ultimately, the appeal to law and law-likeness seems mostly to do rhetorical work, asserting the significance, universality, and fundamental character of phenomena so dubbed rather than demonstrating it.

The remaining chapters of Part I prove more fruitful. Powell explains how instances of convergent evolution might amount to natural experiments, de facto replays of the tape of life that evince robust replicability. He then specifies several criteria that putative instances of convergence must meet if they are to provide evidence for the ubiquity of life and mind in the universe. First, convergent traits must be adaptive, solving design problems that would be pervasive in any history of life. They should also be one of only a "few optimal solutions" to a given design problem, which would be "accessible to selection ... irrespective of the internal constraints of phylogeny" (100). In addition, the characterization of the trait must be specific. So, for example, the wing of a bird might be described as a wing, or a forelimb adapted for flying, or a feathered forelimb adapted for flying. The first description could be applied to both bees and bats as much as birds, the second can applied to bats and birds, but not flying insects, and the third to birds alone. To say that there is convergent evolution for wings is likely true but says something so general as to be trivial and insufficient to support the robust replicability thesis. Convergent traits must also be independent; that is, the trait (or crucial developmental components giving rise to the trait) cannot be shared by the possessors' most recent common ancestor. Because all life on Earth is related and all traits of organisms are to some extent structurally and developmentally connected, independence is always a matter of degree. Nonetheless, the wider the phylogenetic distance between two iterations of the same trait the more a case of convergence supports robust replicability. Finally, if a trait is to be robustly replicable it must obtain under conditions that are fairly common in the universe (149). Together, these criteria supposedly pick out "nontrivial bundles of specific properties that ... exhibit a truly robust range of counterfactual stability" that we might reasonably project to life in the rest of the universe.

Although Gould is positioned as a foil, those familiar with Gould may wonder how much he would really disagree with Powell's position. After all, Gould (1987, Kindle Loc 5227 ff.) not merely supported the SETI (Search for ExtraTerrestrial Intelligence) program but did so on the basis of convergent evolution. Gould $(1980,41)$ believed that convergence is driven by natural selection and that, "while convergence never renders two complex organisms completely identical ... close repeats [can be explained] as optimal adaptations to common problems with few solutions"; a description that meets a number of Powell's criteria for robust replicability. Of 
course, Gould takes a historical approach to natural history, roundly rejecting the idea that the evolution of life on earth can be explained by simple, fundamental biological laws, particularly ones associated with adaptationism. However, Powell's account of laws is sufficiently vague and thin that it is not obvious that Gould would take issue with it.

It's not simply that Gould might have a difficult time recognising Powell's position as particularly antithetical to his own; we may wonder if Gould would recognize Powell's definition of radical contingency as something he would defend. After all, punctuated equilibrium, one of Gould's hobbyhorses, draws attention to the remarkable stability of species over geological time (Gould and Eldridge 1993) and it's difficult to square this with the idea that "a marginal change in some initial condition ... would tend to result in ... [a] radically disparate [outcome]" from what otherwise would have happened.

The issues on which they most substantially part company are ones that clearly motivate Powell but which remain under-discussed until the end of the book-the implications of contingency and convergence for the place of humans in the world. In contrast, Gould tackles this question explicitly, starting Wonderful Life: The Burgess Shale and the Nature of History with a chapter-length discussion of progressivism and narcissistic anthropocentrism, which he believed characterizes not only the popular but the scientific imaginary of evolution. Here he makes the case for the sheer ubiquity of progressivist ideas in biology, which are exemplified by march of progress pictures - that familiar image of individuals walking in a line that typically starts with a nonhuman ape (bizarrely, sometimes not a human ancestor) but always ends with contemporary man. This "is the canonical representation of evolution" (1990, 31, emphasis his). Gould argues that the idea of a single order of life from which humans arise as both inevitable and superior to all the rest is the last vestige of a theological anthropocentrism that is antithetical to contemporary science. As Gould puts it, "physics and astronomy relegated our world to a corner of the cosmos, and biology shifted our status from a simulacrum of God to a naked, upright ape" (44). Although this commitment to dislodging an ideological and unscientific commitment to anthropocentrism is a key motivating consideration for Gould, engagement with this point is absent in Powell's discussion.

Part I concludes with a Coda that briefly addresses the major transitions in evolution, such as the transition to multicellularity and eukaryogenesis. Here, as in the rest of Part I, Powell's effort to articulate a middle ground between his picture of Gould and his critics, seems more like a wavering between the two. We are assured at the end of the Coda that

Radical contingency remains a very real possibility ... for some singular major transitions and key innovations out of which animal forms have been built ... [but] what we can say with confidence is that some specific forms (though not body plans) - and many more generic forms and functions - are robust probably across the evolution of animal grade morphological complexity, quite possibly across the evolution of eukaryote-grade cellular complexity, and perhaps across the evolution of life itself. (160)

Powell's confidence is misplaced. By this point in the book it is not obvious that Powell has shown that there are law-like evolutionary regularities sufficiently specific or independent to guarantee that a rerun the tape of life from the Cambrian-never mind the deep Proterozoic era-would produce beings anything like humans in any significant respect. This is, in effect, the project of the rest of the book.

Part II attempts to marshal the technical tools and criteria explicated in the previous part to assess whether minds that are in some sense humanlike are ubiquitous in the universe. The idea seems to be that even if we have to concede the ground to Gould about the radical contingency 
of body plans, humanlike minds are robustly replicable. As I shall explain in the rest of this paper, despite the significant mass of evidence he brings to the cause, I don't think the project succeeds at all.

Powell begins with an assertion that eyes, or more generally "image forming sensory modalities" (163) are of central importance to mind. "Seeing ... is the evolutionary gateway to more complex forms of cognition and behavior, and even to consciousness itself" (164). Certainly, eyes are a classic and powerful case for convergent evolution, having independently originated in various different taxa and clearly solving a set of pervasive selective problems concerning the acquisition of real-time information from the environment. Both camera-type eyes-found across a striking array of eukaryotes—and compound eyes—typical of arthropods-are bound by the laws of optics, which constrain the eye's function and significantly determine its form. Importantly, Powell is only interested in a subset of eyes, "focus-capable eyes," which, he claims, "in conjunction with sophisticated information processing capabilities, are capable of producing a 'visual scene'" (166), or mental map.

In addition, he insists that a diverse array of sensory capacities, from echolocation to electrolocation, are in fact just varieties of seeing. Of course, Powell needs to shoehorn these very different modalities into a single basic sensory modality to meet his requirements for true convergence, that is, that adaptive solutions to selection pressures are "highly circumscribed" and the description of convergent traits is specific. If these sensory modalities are many different solutions to the same adaptive problem or are only the same thing under some vague description, they fail to count in favour of the robust replicability thesis.

Unfortunately, Powell significantly undersells the true diversity of image-forming capacities, seeming to assume that the integration of sensory modalities in other animals and the sensory modalities themselves will be much as they are in humans. Powell dismisses the possibility that image-forming senses like echolocation, electrolocation or even whiskers may blur into the sense of sound or touch, from the phenomenal perspective of the perceiving organisms. He maintains that all real-time information from waveform energy is much the same (163), ignoring the fact that electric fields and sound reflect and refract through objects in very different ways and will have their own sets of strengths, weaknesses, and illusions. This is to say nothing of the many ways spatial information can be projected into a scene (a topic perhaps best understood in aesthetics [Willatts 1997]) and the many different ways of navigating a physical environment - from beaconing, to route following, to dead reckoning, to using landmarks, to using mental maps (Rescorla 2018). Ultimately, that many organisms have image-forming, sensory modalities is simply not a specific enough description to be an interesting case of convergence. Moreover, it is not clear why there cannot be some highly cognitively sophisticated animals in some environments where touch, sound, or chemical signalling provide information as good, or better, for producing a mental map. The point is not that no other animal has "humanlike" image-forming perceptual capacities-I am not objecting to Powell's anthropomorphism. The point is that many image forming sensory modalities may not be particularly humanlike and the world "seen" through these eyes, ears, whiskers, and (in the case of electrolocation) whole bodies may be unimaginable to us, even as we successfully navigate interactions with the same objects and each other.

Powell then moves from image-forming perceptual mechanisms to the deep, unified structure of experience. In what is perhaps the strangest turn of the book, Powell co-opts Uxeküll's concept of the Umwelt to name this species-specific unified consciousness. While Uxeküll might seem like a kind of ally, given his emphasis on treating individual animals as cognitive subjects, rather than quasi-machines, this is where the allegiance ends. Those familiar with Uxeküll will recognize some of the criticisms of Powell's account of seeing above as resonating

๑ OPEN ACCESS - PTPBIO.ORG 
in an Uxeküllian tone. In fact, even for humans, Uxeküll (2010, 61-70) does not prioritize what he calls "visual space," roughly equivalent to Powell's mental maps, but sets it alongside "effect space" (54-9) and "tactile space," all three of which "interpenetrate and complete but also partially contradict one another" (54). When there are contradictions it is not always (or perhaps even usually) visual space that predominates, as our implicit understanding of the size constancy of objects around us as we move clearly suggests (61-2). Contra Powell, what stands out for Uxeküll is the extraordinary diversity of perception, cognition, and behaviour in the animal kingdom and the remarkably different Umwelts in which different animals live. From this perspective, to say that animal minds are all the same kind of thing makes about as much sense as saying that animal guts are all the same kind of thing. It's not meaningless to talk this way but it is certainly insufficiently specific to count as a nontrivial case of convergence.

To be fair to Powell, he admits his departure from Uxeküll's theory, noting that Uxeküll had no interest in the study of phenomenal experience or consciousness, which, for Kantian reasons, he took to be inaccessible to third-person perspectives (191). Powell goes into some detail, providing an account of the role of consciousness in animal cognition, linking it to the integrated mental maps that place the conscious subject at the center of an Umwelt of objects and the evolution of brains (187-212). However, his claim that consciousness must be unified is simply asserted. Here again, it is difficult to see why we should accept this claim. Hume $(1739 / 2010, \S V I)$ is not the only critic of the idea of a unified consciousness. Large swaths of the Buddhist tradition also reject it and, moreover, take this to be an empirical discovery (Garfield 2015, Kindle Loc. 2572-878). No doubt some degree of cognitive integration some of the time is important to any cognitive animal; but what degree and of what faculties may well vary considerably both across and within species. 1 Of course, this does not imply that no animals other than humans have subjective states (or minds), but it does undermine Powell's assertion that they are all one type of thing, specific enough to ground the robust replicability thesis.

This dubious account of minds is followed by a fascinating overview of the deep evolutionary history of the brain and a discussion of the possibility that brains may have evolved more than once on Earth (213-34). As interesting as the discussion is, the cellular structures of eukaryotes plausibly provide a developmental constraint that undermines any support for the robust replicability thesis. It is not that the general point is wrong. As Peter Godfrey-Smith (2016, 21-6) has pointed out, once an organism has evolved a complex body, it has an internal coordination problem - how to make all its specialized parts work together; and, if it finds itself in a world of risk and opportunity, it has an external coordination problem-first identifying the risks and opportunities and then getting all those parts to avoid or make use of them. If an organism has a single centralized organ that does most of this coordination, that will be, functionally speaking, a brain, but surely this is insufficiently specific to count toward the robust replicability thesis.

The final full chapter is an interesting survey of a huge variety of studies of arthropod cognition (and some research on cephalopods too). Here Powell focuses on the impressive cognitive abilities of bees and jumping spiders. We read of "flexible learning capabilities ... that, in some cases, rival those found in mammals and birds" (240), Gestalt-like perception enabling the recognition of human faces and artistic styles (243-4), "categorization, concept formation, planning, causal reasoning, and social learning" (249), "numerosity competences" (254), and "hunting techniques [that] are eerily reminiscent of the stalking behavior of predatory cats" (255). Despite these impressive capacities, Powell concedes that, "the evidence for so-called cognitive

\footnotetext{
${ }^{1}$ It is worth remembering how much visual information in the human case fails to be integrated into our consciousness, as is evidenced by various examples of inattentional blindness (Simons and Chabris, 1999), or our poor results in some short-term memory tasks when compared with chimpanzees (Inoue and Matsuzawa 2009).
} 
maps in insects is mixed" (256), even for bees. He fails, however, to notice that this seriously weakens his own position - the tight connection between vision, cognition, and unified consciousness on which his claims about the robust replicability of mind rest.

Another peculiarity of this chapter is that Powell slips back and forth between describing bees and jumping spiders and the capacities of insects generally. Arthropods are an enormous and ancient taxon and there are no good reasons to think that the various cognitive capacities exhibited by bees and jumping spiders are widely shared (though some of them may be). Consider Uxeküll's most famous example, the tick. This animal is eyeless and, as Uxeküll (Uxeküll 2010, 50-1) describes them, has an Umwelt limited to the smell of butyric acid, the collision with their prey, the feel of hair free skin, and the warmth of the blood on which they feed, along with an array of reflexes to match-climb, drop, find a hairless patch, feed, and drop again. Here, once again, Gould echoes. Although we often like to think of the march of life as evolving to ever-increasing complexity, most organisms currently alive on our planet-all of whom have ancestors just as ancient and finely sculpted by evolutionary processes as the rest of us-are single-celled organisms. Most contemporary arthropods do not appear to be little hairy geniuses, yet do very well in the evolutionary scheme of things. If there are robust biological laws pushing organisms toward cognitive complexity and consciousness, these must be not just balanced but dwarfed by the power of the biological laws keeping things brainless and/or simple.

Part II ends with another Coda, followed by a brief conclusion. In the Coda we leap from arthropods to Homo sapiens and Powell makes the case, which he admits is all too brief, for our species' uniqueness. He poses the following puzzle: If the universe is ubiquitously populated by minds, why haven't extraterrestrial beings with advanced technology made contact with us? The best explanation, he suggests, is that humans alone have taken the requisite evolutionary path. Against all odds, many contingent accidents of evolution - from opposable thumbs, to symbolic capacity, morality, and more - have come together in just the right way to produce a being capable of cumulative culture and, concomitantly, technology. But, are all and only these traits truly required for either cumulative culture or the development of technology? And are they as rare as Powell suggests? A more complete discussion of other animal taxa-like chordates, perhaps even mammals - might make the gap between humans and all other animal species seem rather narrower. After all, opposable digits or other appendages that do a similar job, like elephant trunks or octopus tentacles, are scattered throughout the animal world. There is also evidence of culture (McGrew 2015; Whitehead and Rendell 2015), socially learned norms of behaviour, and related policing strategies for a number of animal species (Andrews 2020; Bekoff 2019; de Waal 1998), as well as at least rudimentary symbolic capacities (Hobaiter and Byrne 2014). In the endless tinkering and experimentation of evolution, these traits and perhaps others that might also give rise to cumulative culture are likely to be thrown together from time to time. As Gould (1987, Kindle Loc 5231) muses, "perhaps, in another form on another world, intelligence would be as easy to evolve as flight on ours." Moreover, given the extent to which human technology has increasingly shifted from advantageous niche construction to the destruction of our own species' niche (and, indeed, that of chordates and other taxa more generally), we cannot really expect any extraterrestrial that is too much like us to have survived. Here, as in the rest of the book, we find bold assertions and selective reading which will only convince those who already agree with Powell.

In the last pages of the book, Powell shifts to "The Morals of Macroevolution” (279). Here he touts the transcendent implications of convergence and the robust replicability thesis, while painting a rather grim picture of the universe should they turn out to be false. He writes: 
Convergence, if it proves to be a potent force in evolution, could provide a cosmic connectedness-for it is a story about the immense chasms that life will cross to reach the same place .... Radical contingency, should it prevail, would compel us to confront the infinite isolation of a desolate universe, drawing us closer to the only cosmic companions we will ever have: the meaningful minds with whom we share this pale blue dot. (280)

Here, it seems, we discover what the book is really about-humans.

While I applaud Powell's avowed comradery with humanity's fellow earthlings, radical contingency is consistent with both multiple trait-specific patterns of convergence and a populous universe of cognitive beings, who are more or less like humans in an endless variety of different ways. Such beings may surpass humans in some capacities (just as some of our fellow earthlings do) while having other capacities so utterly alien that they largely defy our comprehension. We do not need to appeal to universal biological laws that guarantee that other animals will be drawn in our image to find companionship with them or respect them and their life ways. Indeed, if we are to hope to find less destructive ways of living with the other beings who populate our planet we may need to appreciate their differences rather than demanding that they must be like us to matter to us. We can hardly imagine ourselves ready to meet any extraterrestrial neighbours until we learn to see our kin for who they are and do better by them.

\section{Acknowledgments}

Thanks to an anonymous reviewer for their careful reading as well as Michael Brett-Crowther and my colleagues at Dalhousie University for feedback on previous drafts.

\section{Literature cited}

Andrews, Kristin. 2020. "Naïve Normativity: The Social Foundation of Moral Cognition.” Journal of the American Philosophical Association 6 (1): 36-56.

Bekoff, Marc. 2019. “When Dogs Play, They Follow the Golden Rules of Fairness.” Psychology Today, Accessed July 2, 2020. https://www.psychologytoday.com/us/blog/animal-emotions/201911/ when-dogs-play-they-follow-the-golden-rules-fairness.

Garfield, Jay. 2015. Engaging Buddhism: Why it Matters to Philosophy. Oxford: Oxford University Press.

Godfrey-Smith, Peter. 2016. Other Minds: The Octopus, the Sea, and the Deep Origins of Consciousness. New York: Farrar, Straus, and Giroux.

Godfrey-Smith, Peter. 2009. Darwinian Populations and Natural Selection. Oxford: Oxford University Press.

Gould, Stephen Jay, 1990. Wonderful Life: The Burgess Shale and the Nature of History. New York: W. W. Norton.

Gould, Stephen Jay. 1985/1987. The Flamingo's Smile: Reflections in Natural History. W. W. Norton \& Company. Kindle Edition.

Gould, Stephen Jay. 1980. The Panda's Thumb: More Reflections in Natural History. New York: W. W. Norton and Company.

Gould, Stephen Jay and Niles Eldridge. 1993. "Punctuated Equilibrium Comes of Age." Nature 366: 223-7. 
Hobaiter, Catherine and Richard Byrne. 2014. "The Meanings of Chimpanzee Gestures." Current Biology 24: 1596-1600.

Hume, David. 1739/2010. A Treatise of Human Nature. Project Gutenberg. Accessed July 30, 2020. https://www.gutenberg.org/files/4705/4705-h/4705-h.htm.

Inoue, Sana and Tetsuro Matsuzawa. 2009. "Working Memory of Numerals in Chimpanzees.” Current Biology 17 (23): R1004-1005.

McGrew, William. 2015. “The Cultured Chimpanzee: Nonsense or Breakthrough?” Human Ethology Bulletin-Proc. of the XXII. ISHE Conference: 41-52.

Powell, Russell. 2019. Contingency and Convergence: Toward a Cosmic Biology of Body and Mind. Cambridge: MIT Press.

Rescorla, Michael. 2018. "Maps in the Head?" In The Routledge Handbook of Philosophy of Animal Minds, edited by Kristin Andrews and Jacob Beck. New York: Routledge.

Simons, Daniel and Christopher Chabris. 1999. "Gorillas in Our Midst: Sustained Inattentional Blindness for Dynamic Events.” Perception 28: 1059-1074.

Uxeküll, J. von. 2010. A Foray into the Worlds of Animals and Humans, With a Theory of Meaning, translated by Joseph D. O’Neil. Minneapolis: University of Minnesota Press.

de Waal, Frans. 1998. Chimpanzee Politics: Power and Sex among Apes (Revised). Baltimore: Johns Hopkins University Press.

Whitehead, Hal and Luke Rendell. 2015. The Cultural Lives of Whales and Dolphins. Chicago: University of Chicago Press.

Willats, John. 1997. Art and Representation: New Principles in the Analysis of Pictures. Princeton: Princeton University Press.

(C) 2021 Author(s)

This is an open-access article distributed under the terms of the Creative Commons Attribution 4.0 International license, which permits anyone to download, copy, distribute, display, or adapt the text without asking for permission, provided that the creator(s) are given full credit.

ISSN 2475-3025 